\title{
Lagerplatz Winterthur
}

\author{
Benjamin Zemann, Barbara Buser
}

\section{$1 \quad$ Einleitung}

Das Lagerplatzareal in Winterthur, Schweiz, eignet sich im besonderen Maße als gutes Beispiel für eine Arealentwicklung mit Einbezug der Mieter*innen. Mit der Entwicklung der gesamten Konversionsflächen des ehemaligen Sulzerkonzerns entwickelte sich auf dem Lagerplatz eine spezielle Kultur. Die klassischen Normative und Gesetze der Immobilienökonomie wurden verändert oder sogar außer Kraft gesetzt. Das rund $50.000 \mathrm{~m}^{2}$ große Teilareal wurde 2009 von der Sulzer Immobilien AG an eine Pensionskasse, die Stiftung Abendrot aus Basel, verkauft. Dies gelang auf Initiative der damaligen, in einem Arealverein organisierten Zwischennutzer*innen mit zeitlich beschränkten Verträgen. Mit dem Verkauf an die Stiftung Abendrot wurde der Grundstein für eine nachhaltige Entwicklung des gesamten Areals gelegt. Gemeinsam mit dem Arealverein formulierte die Eigentümerin Perspektiven der zukünftigen Entwicklung. Heute, rund zehn Jahre später, ist ein großer Teil der beschlossenen Entwicklungsmaßnahmen Realität. Das Lagerplatzareal hat sich zu einem lebendigen Stadtquartier entwickelt, dessen Ausstrahlung weit über die Stadtgrenzen hinausreicht.

Der vorliegende Beitrag ist ein Praxisbericht und möchte auch als solcher verstanden werden. Der Schwerpunkt liegt in der Erläuterung der Entwicklung im Kontext partizipativer Entscheidungsmechanismen. Dass die Entwicklung auch unter ökonomischen Aspekten als sehr erfolgreich angesehen werden kann, mag im ersten Augenblick erstaunen.

\section{Sulzerareal Mitte}

Winterthur ist mit gut 100.000 Einwohner*innen eine kleine „Großstadt“ in der Schweiz. Die geschichtliche Entwicklung reicht bis ins Römische Reich zurück. Im 19. Jahrhundert entwickelte sich Winterthur rasch zu einem klassischen Industriestandort. Mit den Firmen Sulzer und Rieter entstand eine metallverarbeitende Industrie, die ihre Produkte in die ganze Welt lieferte. Rieter und v. a. Sulzer prägten von 1950 an das Stadtbild auch mit gezielter Wohnbauförderung. Es entstanden im gesamten Stadtgebiet viele noch heute wertvolle Quartiere. 
Die Ölkrise Mitte der 1970er-Jahre traf auch die Traditionsunternehmen in Winterthur. Mit verschiedenen Reorganisationen und Diversifizierungen des Wertschöpfungsangebots versuchten die Firmen, den drohenden Abbau zu vermeiden. Gelungen ist dies nicht wirklich. Arbeitsplätze wurden ins günstigere Ausland verlegt. Insbesondere Sulzer wurde zu einem Spielball von Finanzinvestoren. Der Entscheid, auf die Produktion von schwerindustriellen Gütern zu verzichten, fiel Ende der 1980er-Jahre.

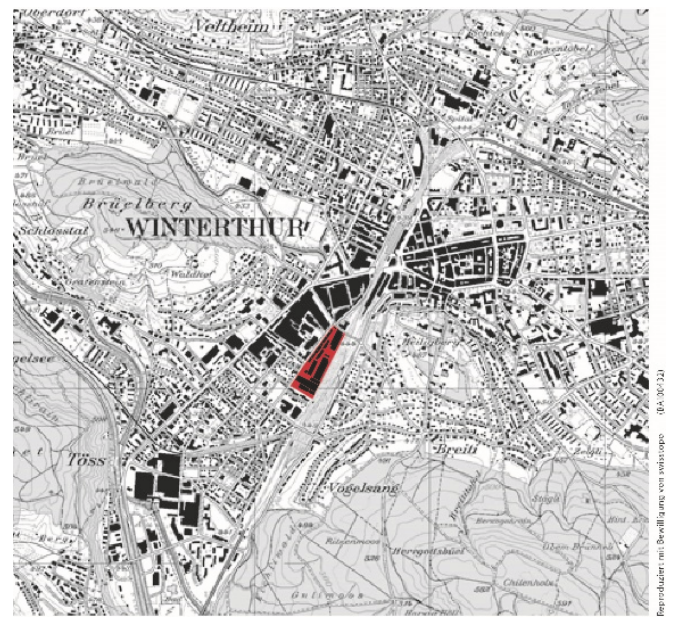

Abbildung 1: $\quad$ Lage Lagerplatzareal

Quelle: Nutzungskonzept Lagerplatz 2010, Arealverein Lagerplatz, S. 31.

Das gesamte Sulzerareal Mitte (vgl. Abb. 1) umfasst ca. 22 ha Land und ist damit fast gleich groß wie die historisch gewachsene Innenstadt von Winterthur.

Auf den Entscheid, die Produktionsstandorte in der Stadt aufzugeben, folgten Jahre der Ideensuche und Planung. Die einst „verbotene Stadt" sollte sich öffnen. $\mathrm{Zu}$ Beginn wollte die Sulzer Immobilien AG, Eigentümerin der Areale, das gesamte Gebiet in einem Wurf neu planen und in eigener Regie überbauen. Ein international ausgeschriebener Architekturwettbewerb wurde von den renommierten Architekten Jean Nouvel, Emanuel Cattani et Associés gewonnen. Einsprachen gegen das Vorhaben verzögerten die Umsetzung. Die Rezession und Immobilienkrise Ende der 1990er-Jahre zwang die Eigentümerin zu einem radikalen Umdenken. 2001 entschied Sulzer Immobilien, keine eigenen Projekte mehr zu entwickeln. Stattdessen wurde das gesamte Areal in vier Teilareale, darunter auch den Lagerplatz, aufgeteilt. Sulzer Immobilien beschränkte sich auf die Aufberei- 
tung der Teilareale und verkaufte diese Schritt für Schritt an zahlungskräftige Investor*innen.

Mit diesem grundlegenden Entscheid ermöglichte die Eigentümerin eine langsamere und bedarfsgerechte Entwicklung der einzelnen Areale. Die Politik und breit abgestützte Initiativen verschiedener Interessengruppen unterstützten den Entscheid. So entstand mitten im Herzen von Winterthur ein Laboratorium für Stadt- und Arealentwicklung. Heute, fast 20 Jahre nach jenem Grundsatzentscheid, ist das Sulzerareal Mitte in Winterthur ein weit über die Kantonsgrenzen hinaus bekanntes Beispiel für eine gelungene Arealentwicklung. Es ist ein lebendiges Stadtquartier entstanden, welches sich nach den Bedürfnissen und durch die unterschiedlichen Aktivitäten der Nutzer*innen stetig weiterentwickelt.

\section{$3 \quad$ Entwicklung des Lagerplatzareals}

Das Lagerplatzareal als Teil des Sulzerareals Mitte lässt sich eindeutig abgrenzen: Die Eisenbahngleise im Norden und die Tössfeldstraße im Süden trennen das Lagerplatzareal vom restlichen Areal Sulzer Mitte. Diese geografischen Voraussetzungen begünstigten eine eigenständige Entwicklung.

Mitte der 1990er-Jahre kaufte die Schweizerische Post einen Teil des Areals mit dem Ziel, ein großes Paketverteilzentrum zu bauen. Nach Jahren des Abwägens entschied sich die Post für einen anderen, zentraler gelegenen Standort. Währenddessen beschloss die Sulzer Immobilien, entgegen ihrer ursprünglichen Absicht, Zwischennutzungen zuzulassen. So entstand innerhalb kürzester Zeit ein buntes Neben- und Durcheinander von Werkstätten, Atelierräumen, Lagern und Freizeitaktivitäten unterschiedlichster Art. Im Jahr 2006 schlossen sich die Zwischennutzer*innen zum Arealverein Lagerplatz zusammen.

2007 beauftragten Sulzer Immobilien und die Stadt Winterthur eine Testplanung. Eine Zonenänderung sollte möglichst viel Wohnfläche erlauben, um das Grundstück gewinnbringend veräußern zu können. Diese Testplanung löste eine heftige Debatte auf verschiedenen Ebenen aus. Insbesondere der neu gegründete Arealverein wollte sich nicht einfach mit der Situation abfinden.

\section{Eigeninitiative auf dem Lagerplatz}

Im Nachhinein sollte sich die Gründung des Arealvereins als Initialzündung für die heutige Entwicklung des Lagerplatzareals herausstellen. Mit der Testplanung und dem Verkauf des Areals drohten die bisherigen Nutzer*innen ihre inzwischen etablierten Räume zu verlieren. Das wollten sie verhindern. Mit tatkräftiger Unterstützung durch den Stadtrat machte sich der Arealverein auf die Suche nach 
möglichen Investor*innen. Über das Gundeldinger Feld und die Kantensprung AG in Basel gelangten sie an die Stiftung Abendrot, eine nachhaltige Pensionskasse mit einschlägiger Erfahrung in der Entwicklung von Industriebrachen. Das Gundeldinger Feld ist ein ehemaliges Industrieareal in Basel, welches vor rund 20 Jahren umgenutzt wurde. Heute wird das Gundeldinger Feld oft als gelungenes Beispiel für eine Umnutzung dargestellt. Die Kantensprung AG ist Eigentümerin des Gundeldinger Felds und die Initiatorin der Entwicklung Gundeldinger Feld. Die Stiftung Abendrot und die Kantensprung AG verbindet seit jeher eine enge $\mathrm{Zu}$ sammenarbeit. Es gelang Abendrot, 2009 einen Kaufvertrag für den gesamten Lagerplatz auszuhandeln.

Mitwirken heißt gleichzeitig auch Verantwortung übernehmen. Die Initiative des Arealvereins, aktiv auf die Suche nach Investor*innen zu gehen, war für den Lagerplatz ein erster wichtiger Schritt. Aus der Perspektive der Stiftung Abendrot war das ein Zeichen, dass die bestehenden Mieter*innen auch in Zukunft auf dem Lagerplatz bleiben wollten.

Die Stiftung Abendrot setzte eine vierköpfige Projektsteuerung ein, welche die Entwicklung planen und schrittweise realisieren sollte. Ein erster Schritt nach der Eigentumsübertragung war es, die zeitlich begrenzten Mietverträge in unbefristete Mietverträge umzuwandeln. Damit wurde ein deutliches Zeichen gesetzt: Die Zwischennutzung war Vergangenheit; die Mieter*innen konnten in ihren Räumen bleiben. In einem nächsten Schritt organisierte die Projektsteuerung eine $\mathrm{Zu}$ kunftswerkstatt zusammen mit dem Arealverein, der Nachbarschaft, den Behörden und anderen Interessierten. Das Ergebnis dieser Zukunftswerkstatt war ein Nutzungskonzept, das die Stoßrichtung der Arealentwicklung definierte. Die bestehenden Gebäudevolumina sollten erhalten und durch sorgfältige Planung ergänzt werden. Notwendige Sanierungen und Renovationen wurden zügig in die Hand genommen (vgl. Abb. 2).

Damit die Stiftung Abendrot ihre Kernaufgabe wahrnehmen kann, muss sie die ihr anvertrauten Pensionskassengelder so anlegen, dass zukünftige Rentenansprüche gedeckt sind und ausbezahlt werden können. Diese Grundvoraussetzung wurde in allen Mitwirkungsprozessen nie infrage gestellt. Dementsprechend wurde der Grad der Mitwirkung insbesondere bei einzelnen Mieter*innen definiert. Als Eigentümerin des Lagerplatzareals trägt die Stiftung Abendrot grundsätzlich jegliche Verantwortung. Das Geschäftsmodell von Abendrot sieht jedoch vor, bestehende und zukünftige Mieter*innen in Entscheidungsprozesse einzubinden. So werden z. B. Renditeerwartungen immer offengelegt und verhandelt. Die finanziellen Auswirkungen auf die Mieten sind so transparent. Dieses Aushandeln zwischen baulichen Maßnahmen und Auswirkungen auf die zukünftigen Mieten ist ein wesentlicher Bestandteil der Mitwirkung und der Übernahme von Verantwortung auf der Seite der Nutzer*innen. 

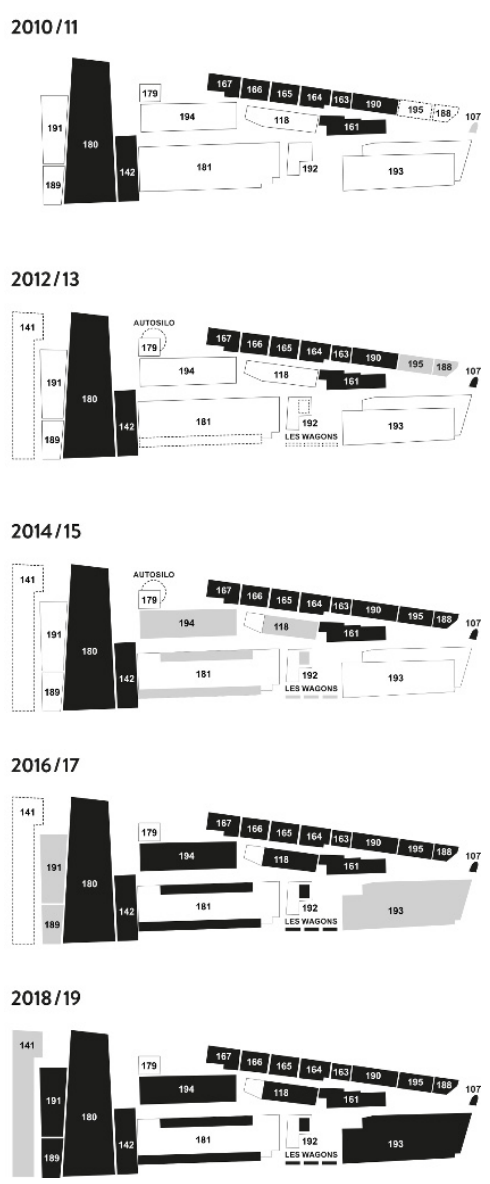

\section{Abbildung 2: Entwicklungsplan}

Quelle: Lagerplatz Winterthur 2010, Stiftung Abendrot, Projektsteuerung Lagerplatz, Einband.

Ein relevanter Aspekt der Mitbestimmung ist es, die gegenseitigen Spielregeln gemeinsam zu vereinbaren. Die Stiftung Abendrot und der Arealverein handelten diese sehr früh aus.

Die nachfolgende Tabelle (vgl. Tab. 1) zeigt die drei Bereiche (Abendrot, Arealverein und Mietende) mit den unterschiedlichen Verantwortlichkeiten bzw. Mitsprachemöglichkeiten auf. Im Bereich A liegen die Verantwortungen ausschließlich bei der Stiftung Abendrot. In den Bereichen B und C wird die Mitwirkung stufen- und phasengerecht definiert. Diese relativ einfach gehaltene Tabelle 
schafft Übersicht und Klärung. Viel mehr ist auf dem Lagerplatz nicht notwendig, um wirkungsvolle Partizipation zu leben.

\section{Tabelle 1: Verantwortlichkeiten Lagerplatz}

\begin{tabular}{|c|c|c|c|}
\hline Themen & $\begin{array}{l}\text { Bereich A } \\
\text { Stiftung Abendrot }\end{array}$ & $\begin{array}{l}\text { Bereich B } \\
\text { Mitsprache } \\
\text { Arealverein }\end{array}$ & $\begin{array}{l}\text { Bereich C } \\
\text { Mitsprache Mieterinnen } \\
\text { und Mieter }\end{array}$ \\
\hline Finanzierung & - & & \\
\hline Nutzung & - & Vorschlagsrecht & $\begin{array}{l}\text { Vorschlagsrecht } \\
\text { pro Gebäude }\end{array}$ \\
\hline Mieterwahl & - & Vorschlagsrecht & $\begin{array}{l}\text { Vorschlagsrecht } \\
\text { für Gebäude }\end{array}$ \\
\hline Verwaltung & - & & \\
\hline Aussenraum & & $\bullet$ & \\
\hline Verkehr, Erschliessung & & $\bullet$ & \\
\hline Kommunikation & & - & \\
\hline Energie & & - & \\
\hline Sanierung der Altlasten & - & & \\
\hline Technische Infrastruktur & • & & $\begin{array}{l}\text { Vorschlagsrecht } \\
\text { pro Gebäude }\end{array}$ \\
\hline Bauliche Massnahmen & $\bullet$ & & \\
\hline
\end{tabular}

Quelle: Nutzungskonzept Lagerplatz 2010, Arealverein Lagerplatz, S. 22.

\section{Akteur*innen}

Die wichtigsten Akteur*innen in der Erfolgsgeschichte des Lagerplatzes waren und sind die damaligen Zwischennutzer*innen. Seit 2000 hatten sie mit ihrer aktiven Präsenz und fantasievollen Umnutzung einzelner Gebäude ein kreatives Biotop geschaffen, das vielen erhaltenswert erschien.

Eine wichtige Rolle spielte auch die Kantensprung AG, die mit ihrem Leuchtturmprojekt Gundeldinger Feld, ebenfalls einer ehemaligen Sulzerfabrik, bereits bewiesen hatte, dass eine neue, gemischte Nutzung der bestehenden Gebäude auch wirtschaftlich rentabel gestaltet werden kann.

Die Stiftung Abendrot kannte als Investorin im Gundeldinger Feld die finanziellen Perspektiven und engagierte sich deshalb auch im Lagerplatz, als alle anderen Investor*innen noch Angst vor solchen Entwicklungen hatten. Dank dem 
Erwerb des Areals durch die Stiftung Abendrot konnten alle Zwischennutzer*innen zu regulären Mieter*innen werden.

Sowohl der Arealverein Lagerplatz als auch der Gesamtstadtrat mit Ernst Wohlwend besuchten innerhalb kurzer Zeit das Gundeldinger Feld in Basel und überzeugten sich von der Machbarkeit der Umnutzung des Bestandes mit sanfter Renovation. So spielte die öffentliche Hand eine große Rolle im Prozess der Vermittlung zwischen Verkäuferin, Käuferin und Nutzer*innen, ohne sich finanziell engagieren zu müssen.

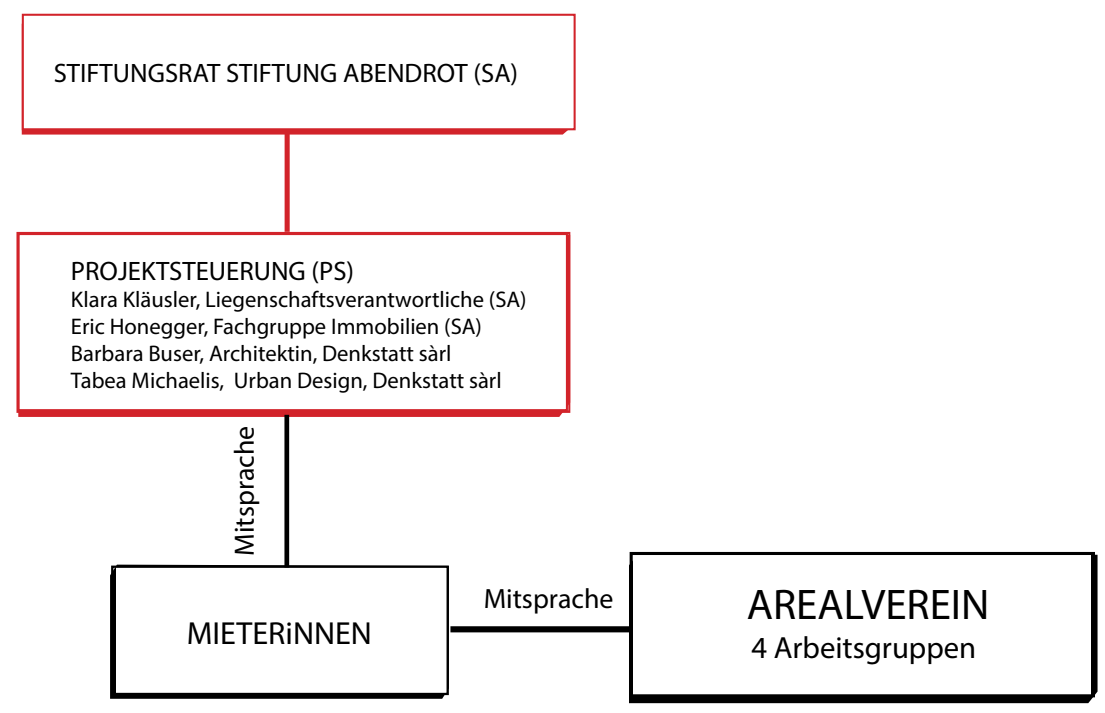

Abbildung 3: $\quad$ Organigramm

Quelle: http://www.lagerplatz.ch/files/243/Lagerplatz_Organigramm-tm.pdf (letzter Zugriff:

20.09.2018), Arealverein Lagerplatz.

Nach dem Vorbild des Gundeldinger Felds schlug der Arealverein vor, das Areal nach dem Kauf im Baurecht zu übernehmen. Die Käuferin Abendrot beschloss aber, das Areal direkt selbst zu bewirtschaften und kein Baurecht zu vergeben. Bei den Verantwortlichen war die Enttäuschung groß, nach dem ersten Schock machte sich aber auch Erleichterung breit. Um die künftige Zusammenarbeit zu definieren, wurde von September bis November 2009 eine Zukunftswerkstatt einberufen. In deren Rahmen wurde auch die zukünftige Mitsprache des Arealvereins definiert (vgl. Abb. 3). 


\subsection{Stiftung Abendrot}

Die Stiftung Abendrot wurde 1984 gegründet und versichert heute über 1.300 Unternehmen, vom kleinen Produktionsbetrieb bis zum großen Dienstleistungsunternehmen. Sie investiert mit Vorliebe in Immobilien, weil diese auch bei Börsenturbulenzen eine stete Rendite garantieren und weil in ihnen die Nachhaltigkeitsvorstellungen von Abendrot eins zu eins umgesetzt werden können. Ihr besonderes Engagement gilt Spezialimmobilien im urbanen Spannungsfeld: der nachhaltigen und stufengerechten Entwicklung und Umnutzung von Industriebrachen, Gewerbebauten und gemischt genutzten Objekten mittels spezifischer Nutzungskonzepte, welche die Quartiersstrukturen, Synergien und einen schonenden Umgang mit Ressourcen fördern.

Als Eigentümerin nimmt die Stiftung Abendrot eine wesentliche Rolle im Partizipationsprozess ein. Sie lässt die Mitwirkung nicht nur zu, sondern hat erkannt, dass diese essenziell zum Erfolg einer Entwicklung beiträgt.

\subsection{Arealverein}

Der Arealverein wurde 2006 von den damaligen Zwischennutzer*innen des Sulzerareals Lagerplatz in einer Notsituation gegründet: Das Areal war zum Verkauf ausgeschrieben worden. Je nach Käufer*in würde die Zwischennutzung früher oder später beendet und die Mieter*innen müssten sich neue Arbeitsorte suchen.

Das Beispiel des Gundeldinger Felds zeigte jedoch auf, dass es auch anders geht: Auch eine sanfte Umnutzung mit einer bunten Mischung verschiedenster Mieter*innen kann wirtschaftlich rentabel sein.

Der Arealverein vereinigt ca. $80 \%$ der Mieter*innen und ist damit als Vertreter der Mieterschaft legitimiert. 2006 wurde eine „Kriegskasse“ geöffnet, um ein professionelles Dossier mit einer alternativen Sicht der Entwicklungsperspektive des Lagerplatzareals zu erstellen. Dies erleichterte die Suche nach einem alternativen Investor bzw. einer alternativen Investorin.

Der Arealverein hat die Grundlage für die Entwicklung auf dem Lagerplatz gelegt. Seine Mitglieder sind die Initianten des sozialen und physischen Gebildes „Lagerplatz“. Sie hatten und haben noch heute den Mut, Ideen neu zu denken, sich vorzustellen, was sein könnte. Aber auch die gegenwärtigen, vielfach organisatorischen Aufgaben gehören zu den Aufgabenfeldern des Arealvereins. Er ist Mittler zwischen den Mieter*innen sowie der Stiftung Abendrot. 


\subsection{Mieter*innen}

Mieter*innen sind, wie bereits mehrfach erwähnt, die wichtigsten Beteiligten an der erfolgreichen Entwicklung des Lagerplatzareals. Im Folgenden werden einige ausgewählte Beispiele dargestellt und kurz erläutert. Damit soll auch die Vielfalt der Nutzungen aufgezeigt werden.

\section{- Beispiel Portier}

Die ehemalige Portierloge war zu einem schmuddeligen Kiosk verkommen. Um sowohl den Neubeginn als auch den Eingang zum Areal zu markieren, wurde der wunderschöne, elegante Baukörper mit dem geschwungenen Dach zu einem kleinen, aber feinen Café ausgebaut (vgl. Abb. 4). Die Cafébetreiber*innen übernehmen die Funktion einer Auskunfts- und Anlaufstelle für das Areal. Sie wissen Bescheid über die Adressen der Mieter*innen und können Besucher*innen einen Arealplan geben. Der Portier ist auch der Treffpunkt für Arealführungen.

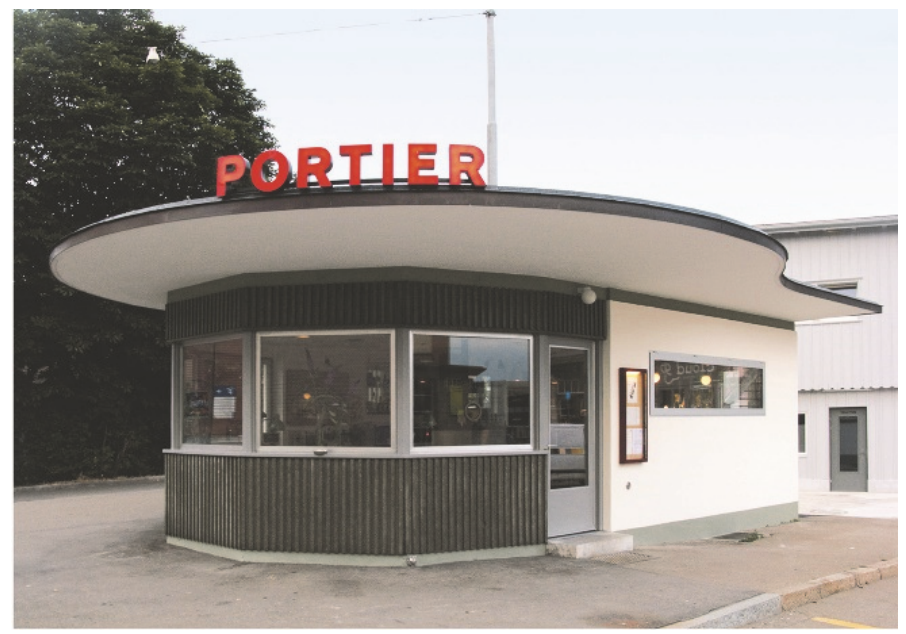

Abbildung 4: Portier

Foto: Lagerplatz Winterthur Foto: Stiftung Abendrot, Vanessa Püntener, Winterthur.

Um die ehemalige Portierloge zu der kleinen Perle zu entwickeln, waren gute Ideen und viel Kreativität gefragt. Die Betreiber*innen waren stark in diesen Prozess mit eingebunden; die Gestaltung des Innenraums haben sie selbstständig geplant und eigenhändig ausgeführt. 
- Beispiel Zürcher Hochschule für angewandte Wissenschaften (zhaw)

Die Umnutzung der ehemaligen Kesselschmiede in der Halle 180 zu einer Architekturhochschule war 1990 einer der ersten Transformationsschritte auf dem Areal (vgl. Abb. 5). Generationen von Architekt*innen wurden seither in der alten Industriehalle ausgebildet. 2012 wurden die Verträge um 20 Jahre verlängert; zusätzlich wurde der Ausbau der Hallen 189/191 für die zhaw beschlossen. Auch der Neubau 141 wird nach seiner geplanten Fertigstellung im 2020 zur Hälfte von der zhaw genutzt.

Die zhaw ist eine wichtige Mieterin verschiedener Gebäude auf dem Lagerplatzareal. Sie wurde als Nutzerin unterschiedlich in die Entscheidungsprozesse eingebunden. Bei den Bauten LP 189/191 und dem Neubau LP 141 war die zhaw von Beginn weg in den Baukommissionen als mitentscheidende Partei vertreten.

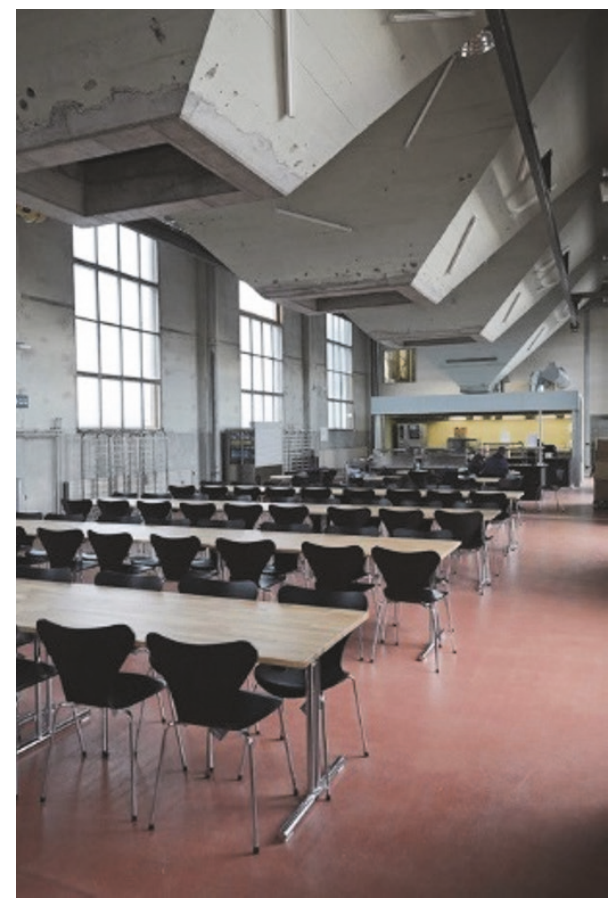

Abbildung 5: Mensa zhaw

Foto: Stiftung Abendrot, Vanessa Püntener, Winterthur. 


\section{- Beispiel Kraftfeld}

Seit 2005 ist das Kraftfeld der kulturelle Treffpunkt des Lagerplatzes (vgl. Abb. 6). Der Club organisiert ein umfangreiches Musikprogramm sowie das jährliche Sonnwendefest. Im Sommer belebt eine Boulebahn den Platz vor dem Feld. Und auch das Bier wird extra für das Kraftfeld gebraut.

Das Kraftfeldteam mietet das Gebäude 161. Dieses befindet sich quasi noch im Urzustand. Auf Wunsch der Mieter*innen beschränkt sich Abendrot auf die normalen Unterhaltsarbeiten und plant keine Gesamtsanierung der Hülle. So bleibt auch die Miete sehr günstig.

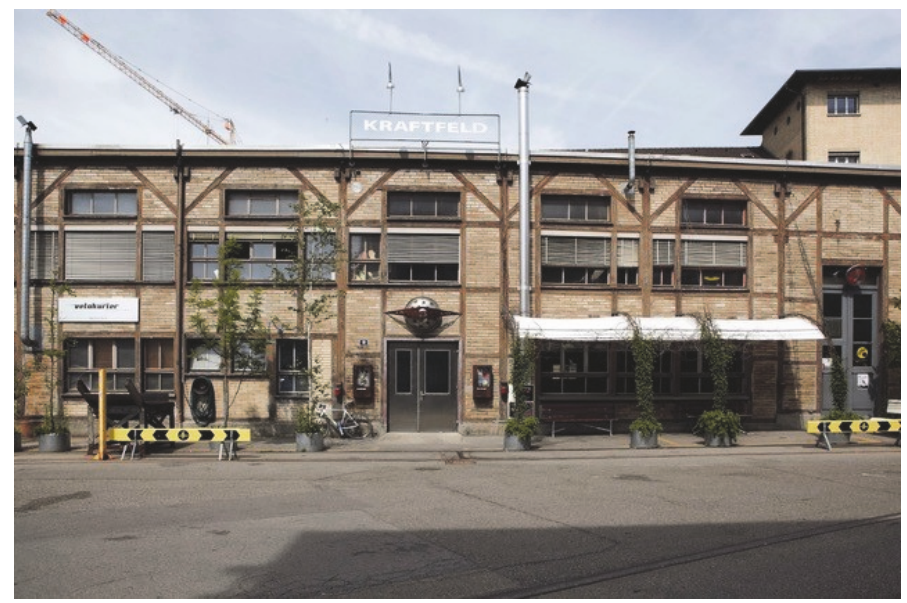

Abbildung 6: $\quad$ Kraftfeld

Foto: Stiftung Abendrot, Vanessa Püntener, Winterthur.

- $\quad$ Valerie Waibel

Die Architektin Valerie Waibel hatte sich als Präsidentin des Arealvereins mit aller Kraft für den Erhalt des Lagerplatzes eingesetzt. Ihr Büro hat sie seit 2005 am Lagerplatz 6. Im selben Gebäude richtete sie für die Projektsteuerung ein Büro ein, im obersten Stock, direkt neben dem Liftmotorenraum, mit Rundumsicht über das Areal. Als nächstes wurde Valerie Waibel mit dem Umbau der kleinen Portierloge in ein Café beauftragt und danach mit der Aufgabe, das Backpacker Hostel oberhalb des Australian Outback einzubauen. Später wurde sie dann damit betraut, die Sanierung der Halle 193 zum Skillspark zu planen und zu realisieren. 
Auch Valerie Waibel gehörte zu den Pionier*innen des Lagerplatzes und war als langjährige Präsidentin des Arealvereins maßgeblich an dessen Entstehung und Entwicklung beteiligt. Mit ihrem Architekturbüro bearbeitet sie kleine und größere Projekte auf dem Lagerplatz, immer in enger Zusammenarbeit mit den Mieter*innen, die dabei ein Mitbestimmungsrecht haben. Denn die Miete steigt und fällt mit den Ansprüchen bzw. den gewünschten baulichen Maßnahmen.

\section{- Beispiel Les Wagons}

Im November 2015 eröffnet, ist das Restaurant Les Wagons ein Nachfolgeprojekt des Café Portier. Die Gleise entlang des Arealplatzes riefen geradezu nach einer neuen arealgerechten Nutzung. Die Idee, drei alte Wagen der Uetlibergbahn als Bistro auf dem zentralen Arealplatz einzurichten (vgl. Abb. 7), kam wie gerufen. In liebevoller Kleinarbeit restaurierten Anja Holenstein und Florian Moser-Dubs die Wagen und bauten sie zu einem Restaurant um. Sie ergänzten die „Station Lagerplatz" mit einem Perron und dem Dach, das auf dem Areal bereits vorhanden war und umplatziert werden musste. Die Zugkomposition bildet einen stimmigen Abschluss des Platzes zum Gleisfeld hin.

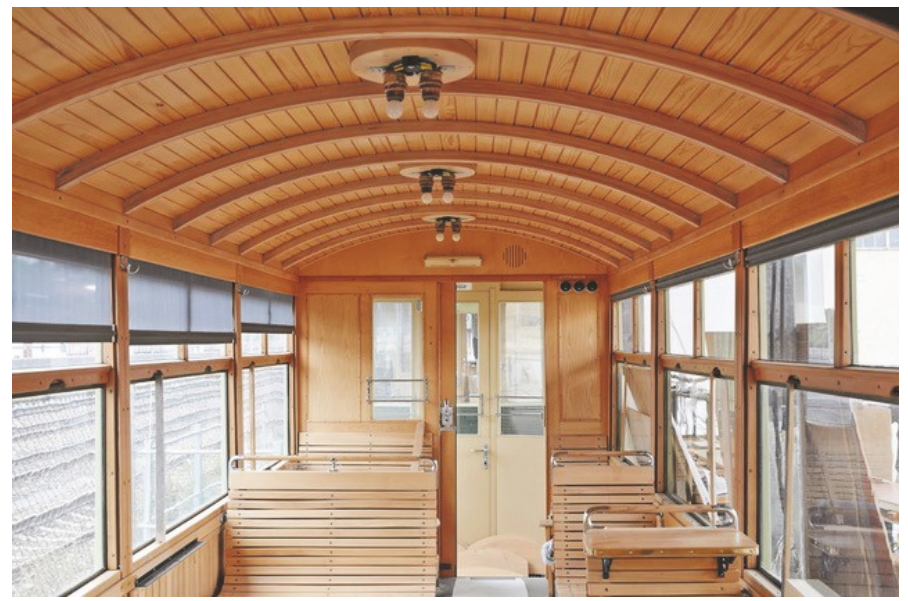

Abbildung 7: $\quad$ Les Wagons

Foto: Stiftung Abendrot, Vanessa Püntener, Winterthur.

Zur Realisierung des ungewöhnlichen Vorhabens war eine enge Zusammenarbeit zwischen Les Waggons und der Stiftung Abendrot nötig. Für die Bahn wurde ein langfristiger Mietvertrag über die Standfläche der Bahn abgeschlossen. Baugesuch 
und Gastrobewilligung wurden von Les Wagons selbst beantragt. Die Restauration der Wagen und deren Umbau zum Bistro wurde ebenso vollständig von den Initiant*innen getragen.

\section{- Beispiel Skillspark}

Roger Rinderknecht und seine Partnerin Edina Banyowski träumten von einer großen Trendsporthalle mit einem breiten Bewegungsangebot für Menschen von einem bis 100 Jahren. Es erwies sich, dass die Halle 193 dafür sehr gut geeignet wäre. Bald konnte mit Abendrot eine Absichtserklärung für den Ausbau der Halle 193 zum Skillspark unterschrieben werden.

Der Skillspark bietet eine BMX-Absprungrampe, 34 Trampoline, Skaterpark, Skaterbowl, Krafttraining und vieles mehr. Das Selbstbedienungsrestaurant mit seinen frisch gekochten Menüs wird von allen Mieter*innen auf dem Lagerplatz geschätzt. Vom Eröffnungstag an wird der Skillspark von Besucher*innen so überrannt, dass die Parkplatzaufsicht massiv verstärkt werden musste.

Der Skillspark ist eines der großen Projekte auf dem Lagerplatz. Von Beginn an waren Edina und Roger gleichberechtigte Mitglieder in der Baukommission und konnten über die Gestaltung, die Materialwahl und die Farbgebung mitbestimmen. Da sich die Miete aus einer Grundmiete für die Halle im Ist-Zustand sowie der Verzinsung der Umbaukosten zusammensetzt, führen Einsparungen direkt zu Mietreduktionen. So wurden dank der Mitbestimmung kostengünstige Lösungen gewählt, die ohne Absprache für die zukünftigen Mieter*innen nicht akzeptabel wären. Die Miete wurde erst nach Genehmigung der Bauabrechnung definitiv festgelegt.

\section{- Beispiel Zusammen_h_alt}

Schon 2012 kam eine Delegation der Genossenschaft Zusammen_h_alt auf die Stiftung zu und erkundigte sich nach den Möglichkeiten eines Wohnungsprojektes auf dem Lagerplatz. Gleichzeitig meldete die bisherige Mieterin zhaw weiteren Platzbedarf an. So entstand die Idee, auf dem einzigen noch unbebauten Baufeld einen kombinierten Schul- und Wohnhausbau zu realisieren, dies auch als Übergang zum sich anschließenden Wohnquartier.

Die Vorstellungen wurden konkretisiert und die Stiftung Abendrot schrieb einen Architekturwettbewerb aus, den Beat Rothen Architekten gewannen. Der Spatenstich fand 2017 statt; 2020 soll der Neubau bezugsbereit sein: Über einem zweistöckigen Sockel von Labors und Schulzimmern entstehen 80 altersgerechte Wohnungen mit großen, gemeinsam nutzbaren Räumen und Terrassen.

Die Mietverträge mit der zhaw und mit Zusammen_h_alt wurden vor Baubeginn unterschrieben. Die Genossenschaft erhielt einen Globalmietvertrag für die 80 Wohnungen auf 20 Jahre. Die Berechnung der Mieten aufgrund der Baukosten 
und des Landwertes ist transparent und wurde von allen Partner*innen akzeptiert. Sowohl die zhaw als auch die Zusammen_h_alt sind gleichberechtigte Mitglieder der Baukommission: Sie entscheiden mit über die Gestaltung, Materialisierung, Farbgebung etc. und tragen auch die finanziellen Konsequenzen der Entscheide in Form von höheren oder niedrigeren Mieten.

\section{Bedeutung für das Quartier}

Die Auswirkungen lassen sich in vier Themenbereiche gliedern:

\subsection{Soziale Plastik}

Für die Stiftung Abendrot ist die Realisierung des Lagerplatzareals eine wichtige Investition, um ihre eigentliche Kernaufgabe, die Verwaltung von Pensionskassengeldern und die Auszahlung zukünftiger Rentenansprüche, zu gewährleisten. Die Eigeninitiative der damaligen Zwischennutzer*innen und die Übernahme von Verantwortung durch die Mieter*innen in verschiedenen Bereichen machen den Lagerplatz zu einer nachhaltigen Investition. Das Areal ist aber nicht nur ein nachhaltiges Bauwerk, sondern auch - frei nach Joseph Beuys - eine „,soziale Plastik“, denn die soziale Struktur, die der physischen, baulichen Struktur überlagert ist, ist gleichzeitig wiederum deren Basis.

\subsection{Bauliche Maßnahmen}

Für die Mieter*innen konnten Räumlichkeiten geschaffen werden, welche den Bedürfnissen derselben entsprechen. Die Zufriedenheit der Mieter*innen äußert sich in geringen Leerstandsquoten und wenig Mieterwechseln. Auch hier steht die Langfristigkeit im Vordergrund. Trotzdem verändern sich Gebäude und das Areal im Laufe der Zeit. Neue Ansprüche und unterschiedliche Vorstellungen müssen diskutiert, Lösungen gefunden werden. Dies geschieht in monatlichen Sitzungen der Projektsteuerung mit dem Arealverein.

Ein Beispiel für eine konstruktive Lösungsfindung ist die Fenstersanierung des Gebäudes LP 190 (vgl. Abb. 8). 


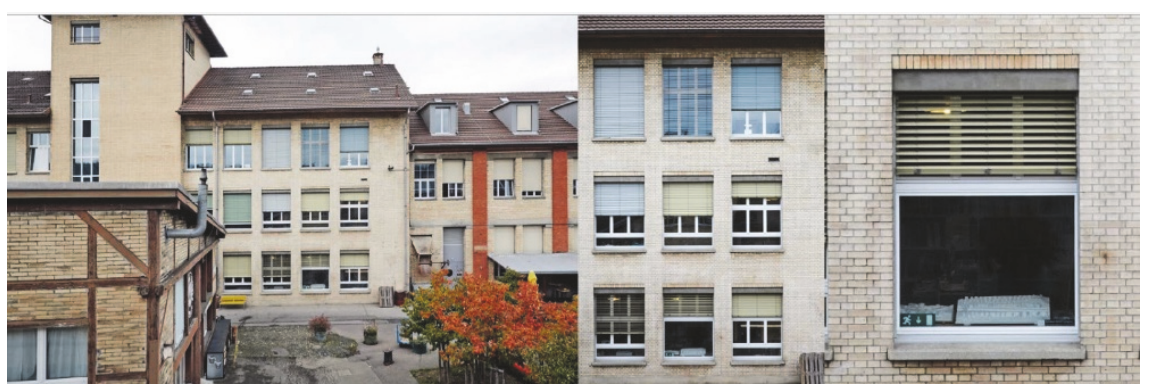

Abbildung 8: $\quad$ Fassadenausschnitt Gebäude LP 190

Foto: Stiftung Abendrot, Vanessa Püntener, Winterthur.

Die rund 60 Fenster des Gebäudes mussten saniert werden. Gemäß Mitspracheregelung konnten die Mieter*innen des Gebäudes bei der Gestaltung mitreden. Die verschiedenen Architekten konnten sich jedoch nicht auf eine Form einigen. Die Projektsteuerung entschied deshalb, dass jedes Büro die Fenster bekommen soll, die es wünscht. Und auch die Farbe der Rafflamellenstoren konnte jede Mieterin und jeder Mieter individuell aus einem Farbfächer auswählen. Kaum eine Passantin und kaum ein Passant realisiert, dass diese architektonisch strenge Fassade durch die verschiedenen Wünsche der Mieter*innen in Form und Farbe aufgelockert wurde!

\subsection{Substanzerhalt}

Eine wesentliche Wirkung der verfolgten Ziele ist der möglichst umfassende Erhalt der bestehenden Bausubstanz. Die ökonomische Bilanz diesbezüglich ist sowohl für die Stiftung Abendrot (langfristige und stabile Rendite) wie auch für die Mieter*innen (kostengünstige Mieten) positiv. Durch den Erhalt der bestehenden Bausubstanz werden riesige Mengen an grauer Energie erhalten und Ressourcen geschont sowie die immateriellen Werte aktiviert. Eine wichtige Voraussetzung dafür ist, dass die Ansprüche an die Flächen und die Nutzung dem jeweiligen Gebäude angepasst werden. Dieses Konzept verlangt viel Flexibilität im Denken sowohl bei den zukünftigen Nutzer*innen als auch bei den Eigentümer*innen.

\subsection{Entwicklung und Belebung}

Das Lagerplatzareal wird als sehr positives Beispiel von Stadtentwicklung wahrgenommen. Während sich bei weitgehend neu gebauten Quartieren die Belebung 
erst nach Dekaden einstellt, konnte das Lagerplatzareal von Beginn an einen lebendigen Charakter entwickeln und behalten. Nutzer*innen blieben auch während der Baumaßnahmen auf dem Areal. Sorgfältige Planung und Kommunikation der einzelnen baulichen Interventionen förderten die Inanspruchnahme.

Ebenfalls erwähnenswert ist die außerordentlich hohe Geschwindigkeit der Entwicklung: Nach nur zehn Jahren ist das Areal umstrukturiert, voll vermietet und belebt. Die Entwicklung soll aber nicht abgeschlossen sein: Neue Anforderungen, Wünsche und neue Gesetze sorgen weiterhin für einen stetigen Wandel.

\section{Partizipation als Erfolgsfaktor}

Die Erfahrungen mit dem Lagerplatzareal haben gezeigt, dass die Mitsprache/Partizipation ein wichtiger Erfolgsfaktor für jede Umnutzung ist. Durch die Mitsprache kommt das ganze Wissen der Anwohner*innen und Interessent*innen, den Expert*innen des Alltags, zusammen und kann für das Projekt fruchtbar gemacht werden. Das Widerstandspotenzial, das bei jeglicher Änderung aktiviert wird, kann beträchtlich reduziert werden. Die Beteiligten können ihre Bedenken äußern und diese werden wahrgenommen. So können Ängste abgebaut werden. Die Eigenleistung und Kreativität der Beteiligten werden gefördert. So entsteht eine lebendige, diversifizierte Umgebung, die so nie geplant werden könnte. Der Genius Loci lässt grüßen! Die Mitsprache bei einem Projekt fördert auch die Eigenverantwortung der Mieter*innen/Akteur*innen: Wer eine Idee selbst vorschlägt, steht auch ganz anders dahinter. Nicht zuletzt ergibt sich ein besserer Zusammenhalt unter Mieter*innen, wenn sie gemeinsam die Mitsprachemöglichkeit wahrnehmen.

Es ist von Vorteil, wenn sowohl die Eigentümer*innen als auch die Projektsteuerung und die Mieter*innen bereits Erfahrung mit Umnutzungen haben. So können Fehler vermieden werden und das Projekt kommt schneller vorwärts. Wichtig ist es auch, der Mitsprache einen klar definierten zeitlichen und finanziellen Spielraum zu gewähren. Die Prozesse folgen nicht unbedingt den vorgegebenen Abläufen.

Es sind unzählige Faktoren, welche den partizipativen Prozess im Zuge einer Arealentwicklung beeinflussen. Für die positive Entwicklung des Lagerplatzes können sechs besonders wichtige Erfolgsfaktoren benannt werden:

- klare Regeln der Mitsprache,

- Verbindlichkeit der Eigentümerin in Bezug auf die Partizipation,

- Entschädigung für Freiwilligenarbeit,

- strukturierte, effiziente Besprechungen,

- demokratische Entscheidungskultur sowie

- positive, faire Konfliktkultur. 
Welche Erfolgsfaktoren letztendlich relevant sind, hängt im Wesentlichen von der bestehenden und zukünftigen Mieterstruktur ab. Letztendlich ist es eine Mischung aus Erfahrungen vergleichbarer Entwicklungen und dem gegenseitigen Aushandeln von Vereinbarungen.

\section{Fazit}

Das Wichtigste vorweg: Die Stiftung Abendrot hätte den Lagerplatz ohne die Initiative des Arealvereins und des Stadtpräsidenten nicht gekauft. Und ohne das konkrete Beispiel des Gundeldinger Felds hätte niemand an die Möglichkeit geglaubt, dass eine solche bunt gemischte Nutzung wirtschaftlich rentabel sein könnte.

Immer wieder wird die Frage gestellt, ob sich dieses Modell auch für andere Arealentwicklungen adaptieren ließe oder ob das Lagerplatzareal einfach ein gelungener Einzelfall sei.

Diese Frage lässt sich einfach beantworten: Das Modell ist erfolgreich adaptierbar, wenn alle Beteiligten das Gleiche wollen und transparente Prozesse vereinbart werden. Selbstverständlich muss das Modell an die jeweilige spezifische Situation angepasst werden. In jeder Stadt, in jeder Gemeinde gibt es brachliegende Gewerbebauten, die nach einer Umnutzung rufen. Sie haben eine Geschichte, man sieht und spürt die Spuren des Gebrauchs, sie sind Identifikationsobjekte der lokalen Bevölkerung. Eigentlich braucht es in jeder Stadt ein Gundeldinger Feld bzw. einen Lagerplatz!

Erfolg hatte dieser dialogische Prozess dadurch, da sich die Architektur den Nutzungen anpasste und nicht die Nutzungen der architektonischen Form. „Form follows function" meint hier, dass das enge An- und Einbinden der Akteur*innen und von deren Bedürfnissen erst die Reaktion und Adaption der lokalspezifischen Strukturen erlaubt. Die Nutzer*innen, verstanden als lokale Expert*innen des Alltags, spielen in diesen Transformationsprozessen eine wichtige Rolle und bieten zeitgleich eine große Ressource an verschiedenen Fähigkeiten und Talenten.

Dass der/die Planer in diesen Prozessen eine moderierende und vermittelnde Rolle zwischen den Beteiligten und den gebauten Strukturen spielen, ist im ersten Moment ungewohnt - zeigt aber in der heutigen Praxis, dass sich in den bauenden Disziplinen neue Aufgaben und Handlungsfelder eröffnen.

Investor*innen wie die Stiftung Abendrot haben den Mehrwert dieser Entwicklungen erkannt. Vermehrt versuchen auch klassisch ausgerichtete Investor*innen partizipative Prozesse zu implementieren, oft mit mäßigem Erfolg. Partizipation muss konsequent geplant und umgesetzt werden. Wenn für alle Beteiligten Win-win-Situationen angestrebt werden und die Anforderungen mit Augenmaß behandelt werden, gelingt Partizipation. Andernfalls wird es sehr schwierig. 


\section{Literatur}

Arealverein Lagerplatz (2010): Nutzungskonzept Lagerplatz. Abrufbar unter: http://www. lagerplatz.ch/entwicklung_245D.html. Letzter Zugriff: 25.3.2018.

Stiftung Abendrot/Projektsteuerung Lagerplatz (2015): Lagerplatz Winterthur (ein Industriequartier im Wandel). Edition Denkstatt. Basel. Abrufbar unter: https://www.denkstattsarl.ch/editions. Letzter Zugriff: 25.3.2018.

Kantensprung AG (2015): Gundeldinger Feld (Dornacherstrasse Basel, Eine Maschinenfabrik im Wandel). Edition Denkstatt. Basel.

Open Access Dieses Kapitel wird unter der Creative Commons Namensnennung 4.0 International Lizenz (http://creativecommons.org/licenses/by/4.0/deed.de) veröffentlicht, welche die Nutzung, Vervielfältigung, Bearbeitung, Verbreitung und Wiedergabe in jeglichem Medium und Format erlaubt, sofern Sie den/die ursprünglichen Autor(en) und die Quelle ordnungsgemäß nennen, einen Link zur Creative Commons Lizenz beifügen und angeben, ob Änderungen vorgenommen wurden.

Die in diesem Kapitel enthaltenen Bilder und sonstiges Drittmaterial unterliegen ebenfalls der genannten Creative Commons Lizenz, sofern sich aus der Abbildungslegende nichts anderes ergibt. Sofern das betreffende Material nicht unter der genannten Creative Commons Lizenz steht und die betreffende Handlung nicht nach gesetzlichen Vorschriften erlaubt ist, ist für die oben aufgeführten Weiterverwendungen des Materials die Einwilligung des jeweiligen Rechteinhabers einzuholen.

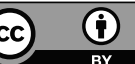

